\title{
Evaluation of hospital acquired infections in the tertiary intensive care unit: a three-year analysis
}

\author{
(D) Fatma Yllmaz Aydın', ๑Emre Aydın² \\ ${ }^{1}$ Dicle University, Faculty of Medicine, Department of Internal Medicine, Diyarbakır, Turkey \\ ${ }^{2}$ Dicle University, Faculty of Medicine, Department of Nephrology, Diyarbakır, Turkey
}

Cite this article as: Y1lmaz Aydın F, Aydın E. Evaluation of hospital acquired infections in the tertiary intensive care unit: a three-year analysis. J Health Sci Med 2022; 5(1): 321-325.

\begin{abstract}
Introduction: Hospital-acquired infections (HAI) occurring in intensive care units (ICUs) are an important risk factor for mortality and morbidity. In some patient groups followed in ICUs, the risk of developing nosocomial infections increases even more. Especially, patients with end-stage renal disease (ESRD) carry a serious risk for HAI when they are hospitalized in ICUs. Our aim is to determine the rate and incidence of hospital infection, the distribution of infections and the most common microorganisms in our ICU, to initiate appropriate empirical treatment and to prevent the development of antibiotic resistance. Material and Method: A total 158 patients with a diagnosis of hospital-acquired infection hospitalized between January 2017 and December 2019 at general internal medicine intensive care unit, were included in this study. The clinical findings, culture results and laboratory data of the patients were recorded. According to years, the HAI rate, density and infection agents in the ICU were determined.

Results: 158 episodes of nosocomial infections were detected in 128 of 556 patients who were hospitalized within three years. The hospitalization day was 9048 , and the three-year ICU HAI rate was calculated as $29.19 \%$. HAI density was 17.45 in 1000 patient days. Bloodstream infection was the most common (30.38\%), followed by ventilator-associated pneumonia (28.48\%) and catheter-related urinary tract infection (24.68\%). Gram-negative microorganisms were the most common infectious agents. Among the Gram-negative bacteria, the most frequently isolated bacteria were A. baumannii, K. pneumoniae and $P$. aeruginosa. Among Gram-positive bacteria, Enterococcus spp. was most frequently isolated

Conclusion: The risk of HAI is high in patients hospitalized in ICUs. In order to control nosocomial infections, HAI incidences and rates should be evaluated, infectious agents, and prospective effective infection control strategies should be developed by taking necessary precautions according to surveillance results. These measures will significantly reduce the incidence of HAI.

Keywords: Intensive care units, hospital infections, gram negative bacteria, central venous catheter, urinary tract, catheter related infections, infection control
\end{abstract}

\section{INTRODUCTION}

Hospital-acquired infections (HAI) are defined as infections that develop 48-72 hours after hospital admission or within 10 days after discharge, in which the patient does not have any infection before admission to the hospital or in the first days of hospitalization (1). HAI occurring in intensive care units (ICUs) are an important risk factor for mortality and morbidity. The incidence of hospital-acquired infections is 2-5 times higher in ICUs than in clinical services. (2). The reasons for this, we can count the patient-related comorbidities such as long hospital stays in ICUs, perform of many invasive procedures, cross contamination and diabetes mellitus, kidney failure, heart failure, and liver failure (3). In some patient groups followed in ICUs, the risk of developing nosocomial infections increases even more. Especially, patients with end-stage renal disease (ESRD) carry a serious risk for HAI when they are hospitalized in ICUs. It is known that these patients have additional comorbid conditions, invasive vascular procedures used for Renal Replacement Therapy (RRT), uremic toxicity and anemia increase the susceptibility to hospital-acquired infections (4).

Hospital infection rates and incidence, foci of infection, causative microorganisms and antibiotic resistance may differ between different ICUs of the same hospital. It is very important for each unit to know its own flora 
and antibiotic resistance, in order to determine the infection control measures to be taken in these units and the appropriate empirical antibiotic therapy. In this study, the epidemiological characteristics of HAIs in the Internal Medicine ICU and their change over the years were investigated. Our aim is to determine the rate and incidence of hospital infection, the distribution of infections by regions and the most common microorganisms in our ICU, to initiate appropriate empirical treatment and to prevent the development of antibiotic resistance.

\section{MATERIAL AND METHOD}

Approval for the study was granted by the Ethics Committee of Dicle University Faculty of Medicine (Date: 06.02.2020, Decision No: 144). All procedures were carried out in accordance with the ethical rules and the principles of the Declaration of Helsinki.

Between January 2017 and December 2019, 556 patients were hospitalized in the general internal medicine intensive care unit. The information of these patients was analyzed retrospectively. Among these patients, regardless of gender, 158 patients aged over 18 years and followed up in the intensive care unit for at least 72 hours, were diagnosed with HAI and included in the study. Patients' HAIs are followed by an active, prospective surveillance study based on patient and laboratory, by infection control doctors and nurses five days a week. This information is recorded daily in the surveillance network. Infections occurring 48 hours after admission to the ICU, but not before or during admission, were defined as HAI. Diagnoses of ventilator-associated pneumonia, catheter-associated urinary tract infection, laboratory-confirmed bloodstream infection, and central venous catheter (CVC) related infection were defined according to Centers for Disease Control and Prevention (CDC) guidelines. (5) In the diagnosis of HAI, peripheral blood as a laboratory finding; leukocyte, platelet count, $\mathrm{C}$-reactive protein (CRP) value, in urine analysis; pyuria and nitrite positivity, radiologically; detection of new infiltration in the lungs, as a clinical finding; parameters such as fever, lung aural finding, hypotension, dysuria and suprapubic tenderness were used. At the time of diagnosis of HAI, clinical and laboratory findings were examined. Cultures of the areas thought to be the focus of infection (urine, sputum, wound site, catheter, and deep endotracheal aspirate from those who received ventilator therapy) were taken from all patients who were thought to have HAI. BACTEC peds plus/F (BD,Sparks, MD) ${ }^{\oplus}$ culture bottles were used for blood samples. Identification of the microorganism and determination of antibiotic susceptibility were performed using the automated Phoenix culture system in line with the recommendations of the Clinical and Laboratory Standards Institute in the USA. The clinical findings, culture results, radiological and laboratory data of the patients diagnosed with HAI were recorded in the prepared standard form. HAI rate and incidence density were calculated. (HAI rate (\%) $=$ Number of HAI/number of patients admitted x 100, Incidence density $=$ Number of HAI/patient days $\mathrm{x} 1000$ ). According to these data, the HAI rate and infection factors in the ICU were determined.

\section{RESULTS}

158 episodes of nosocomial infections were detected in 128 of 556 patients who were hospitalized in the General Internal Medicine ICU between January 2017 and December 2019 and followed up for at least 72 hours. The mean age of 128 patients diagnosed with HAI was 49.6 (18-89). 43\% (n:55) of the patients were female and $57 \%$ (n:73) were male. The hospitalization day was 9048, and the three-year ICU HAI rate was calculated as $29.19 \%$. The incidence of HAI by years was $51.7 \%$, $33.1 \%$ and $11.36 \%$, respectively. HAI density was 17.45 in 1000 patient days. The distribution of hospital-acquired infection rate by years is shown in Table 1 .

Table 1. The number of patients, hospital days, the number of patients who developed HAI, the number of HAI, the rate and density of HAI in the General Internal Medicine ICU between

\begin{tabular}{cccccc} 
Year & $\begin{array}{c}\text { Number } \\
\text { of } \\
\text { inpatients }\end{array}$ & $\begin{array}{c}\text { Hospitalization } \\
\text { day }\end{array}$ & $\begin{array}{c}\text { Number } \\
\text { of patients } \\
\text { who } \\
\text { developed } \\
\text { HAI }\end{array}$ & $\begin{array}{c}\text { HAI } \\
\text { rate }\end{array}$ & $\begin{array}{c}\text { HAI } \\
\text { density }\end{array}$ \\
\hline 2017 & 159 & 2900 & 82 & 51.57 & 28.28 \\
2018 & 142 & 3086 & 47 & 33.1 & 15.23 \\
2019 & 255 & 3062 & 29 & 11.36 & 9.47 \\
Total & 556 & 9048 & 158 & 29.19 & 17.45 \\
\hline & & & & & \\
\hline
\end{tabular}

When we analyzed the three-year invasive deviceassociated infection data, the rate of mechanical ventilator use in ICU patients was 0.65, the day of use was 3908, and the rate of VAP (ventilator associated pneumonia) was $10.24 \%$. The rate of urinary catheter use was 0.99 , the day of use was 8953, and the rate of catheter-related urinary tract infection was $4.36 \%$. The CVC usage rate was 0.8 , the day of use was 7214 , and the CVC-related bloodstream infection rate was 3.05\%.

When the distribution of HAI attacks was examined, bloodstream infection was the first and pneumonia was the second (Table 2). When analyzed by years, the most common cause of infection was catheter-related urinary tract infection in 2017, pneumonia in 2018, and CVCrelated bloodstream infection in 2019. In addition, when we analyzed HD patients by years, we found that the most common CVC-related bloodstream infection developed in these patients for three years. 


\begin{tabular}{|lcccc|}
$\begin{array}{l}\text { Table 2. Distribution of hospital acquired infections according } \\
\text { to the infection sites }\end{array}$ & $\begin{array}{c}\text { Number } \\
\text { of } \\
\text { infections }\end{array}$ & $\begin{array}{c}\text { Percent } \\
\text { of total } \\
\text { infections }\end{array}$ & $\begin{array}{c}\text { HAI } \\
\text { rate }\end{array}$ & $\begin{array}{c}\text { HAI } \\
\text { dansite }\end{array}$ \\
\hline Type of HAI & 48 & 30.38 & 8.87 & 5.31 \\
\hline $\begin{array}{l}\text { Bloodstream infection } \\
\begin{array}{l}\text { Ventilator-associated } \\
\text { pneumonia }\end{array}\end{array}$ & 45 & 28.48 & 8.32 & 4.97 \\
$\begin{array}{l}\text { Urinary catheter related } \\
\text { infection }\end{array}$ & 39 & 24.68 & 7.20 & 4.31 \\
$\begin{array}{l}\text { Central venous catheter } \\
\text { associated bloodstream } \\
\text { infection }\end{array}$ & 22 & 13.92 & 4.07 & 2.43 \\
$\begin{array}{l}\text { Skin and soft tissue } \\
\text { infection }\end{array}$ & 4 & 2.53 & 0.74 & 0.44 \\
Total & 158 & 100 & 29.19 & 17.45 \\
\hline
\end{tabular}

Gram-negative microorganisms were frequently isolated as infectious agents in our ICU. Among the Gramnegative bacteria, the most frequently isolated bacteria were $A$. baumannii, $K$. pneumoniae and $P$. aeruginosa. Among Gram-positive bacteria, Enterococcus spp. was most frequently isolated (Figure 1). When the foci of infection were examined, Klebsiella spp. in bloodstream infections, Acinetobacter spp. in VAP, Klebsiella spp. in urinary catheter-related infections, Klebsiella spp. and Acinetobacter spp. in CVC-related infections were found to be the most common agents. When the antibiotic susceptibility was examined, the most effective antibiotic in $A$. baumanniithat is the most frequent microorganism, was colistin, while its sensitivity was $75.09 \%$ for three years. The most effective antibiotics against other $\mathrm{Gr}$ (-) microorganisms were amikacin, imipenem, and meropenem, while linezolid and vancomycin were effective against $\mathrm{Gr}(+)$ microorganisms.

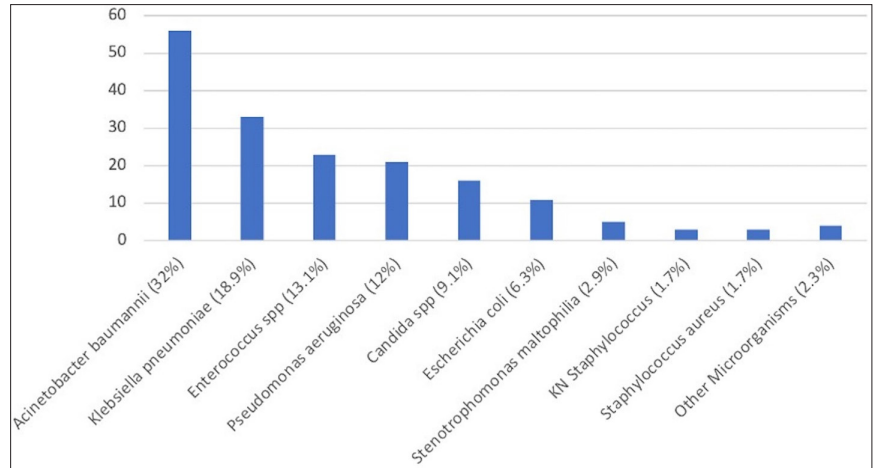

Figure 1. Microorganisms isolated from patients with positive culture

\section{DISCUSSION}

ICUs are units that have many risk factors for the development of HAI, and where invasive procedures such as mechanical ventilator, tracheostomy and catheter are frequently performed. The most important factors that increase the risk of HAI are factors belonging to the patient, invasive procedures, and cross contamination. As the number of invasive procedures increases, the number of mechanical ventilation-associated pneumonia, catheter-related urinary tract infections and CVC-related bloodstream infections increase in parallel. HAI is an important cause of mortality and morbidity for patients followed in the ICU. Therefore, it is important to determine HAI agents and their antibiotic susceptibility in terms of reducing mortality and morbidity.

In different studies conducted in our country, it has been reported that the rate of HAI is between $5.3-64.6 \%$, although it varies according to ICUs (6). When studies on nosocomial infections in the literature were scanned, the rate of HAI was found to be $34.5 \%$ in a prevalence study in which 1150 ICUs from 88 countries participated (7). In their three-year analysis, Taş et al. (8) found the highest HAI rate to be $34.31 \%$ and the lowest to be $13.14 \%$. In another study, 5204 intensive care patients were screened, and 269 nosocomial infections were detected. In this study, the HAI rate was calculated as $27.3 \%$ (9). In our study, we found the HAI rate to be $29.19 \%$ on average, although it decreased over the years. The reason for the low rate of HAI over the years can be counted as active surveillance, attention to isolation measures, especially standard precautions, and the continuity of the education to raise awareness of all staff on this issue. The density of HAI is as important as its rate. In studies conducted in our country, HAI density varies between 15.4 and 44.7 (10). Cetin et al. (11) found the HAI density of 21.9 in their two-year analysis of patients hospitalized in the ICU. In our study, we found the HAI density as 17.45. Our results are compatible with the literature.

Invasive devices increase the risk of nosocomial infection because it disrupts the barrier integrity of the body, facilitates the formation of biofilm, and can cause infection with less bacteria as well as strains with low virulence (10). The widespread use of intravascular catheters in ICUs is one of the most important factors contributing to the development of catheter-related infections. Especially CVCs are the most common types of catheters that can cause these infections (3). When we examine the rate of invasive catheter-related infections and catheter use rates in the literature, according to the National Health Service-Related Infections Surveillance Network of our country's Ministry of Health 2020 data, the rate of mechanical ventilator use in internal medicine ICUs in all university hospitals is 0.46 , the rate of VAP is $8.3 \%$, the rate of urinary catheter use is 0.95 , the rate of catheter-related urinary tract infection is $1.3 \%$, the rate of CVC use is 0.71, the rate of CVC-related bloodstream infection is 3.1\% (12). Sahin et al. (10) found that the rate of VAP in the neurology ICU was $12.4 \%$, the rate of catheter-related urinary tract infection was $2.53 \%$, and the rate of CVC-related bloodstream infection was $5.14 \%$. In another study that included internal and surgical ICUs, the infection rate was found to be 0.39 in 
patients diagnosed with catheter-related urinary tract infection (13). According to our findings, the rate of mechanical ventilator use is 0.65 and the rate of VAP is $10.24 \%$. The rate of urinary catheter use was 0.99 , and the rate of catheter-related urinary tract infection was $4.36 \%$. CVC usage rate was 0.8 , CVC-related bloodstream infection rate was $3.05 \%$. The reason why our VAP rate is higher than our country's data can be attributed to the higher ventilator usage rate. Our rate of CVCrelated bloodstream infection is similar to the data of our country.

Nosocomial infections in ICUs often develop in areas such as the respiratory tract, abdomen, catheter-related bloodstream, urinary system, skin-soft tissue, and central nervous system. Vincent et al. (7) showed in the prevalence study that the most common infections were pneumonia and bloodstream infections. In the study of Taş et al. (8) urinary system infections were the first, while bloodstream infections were the second. In another study, while ventilator-associated pneumonia was the most common HAI, it was followed by central venous catheter-related bloodstream infection (9). In our study, bloodstream infection was the first, while pneumonia was the second. In addition, when we analyzed the infection foci by years, the most common infection foci were catheter-related urinary tract infection in 2017, pneumonia in 2018, and central venous catheter-related bloodstream infection in 2019.

Nosocomial infections are common in hemodialysis patients followed in ICUs. Catheters used especially for RRT constitute an important risk factor for HAI. It is also known that uremia causes immune dysfunction and increases susceptibility to infection (14). In a study examining nosocomial infections in $\mathrm{HD}$ patients, urinary catheter-related infection was the most common, followed by catheter-related bloodstream infection and pneumonia (4). In another study in which 110 cases were evaluated, pneumonia, catheterrelated bloodstream infections and urinary catheterrelated infections were found to be the most common, respectively (15). In our study, the most common central venous catheter-related bloodstream infection was seen in patients undergoing HD.

HAI rates with Gram-negative bacteria, which do not cause infection in healthy individuals but are frequently encountered in ICUs, have increased in recent years $(3,8,9,11)$. When the distribution of causative microorganisms in HAIs in our country is examined, Klebsiella spp. in bloodstream infections, Acinetobacter spp. in VAP, Klebsiella spp. in urinary catheter-related infections, and Klebsiella spp. and Acinetobacter spp. in central venous catheter-related bloodstream infections are the most common agents (16). Şahin et al. found that Acinetobacter spp. in VAP and Klebsiella spp. and E. coli in catheter-related urinary tract infections were the most common agents in their study (10). In a multicenter study, culture results were examined, and Gram (-) microorganisms were detected with $70.7 \%$ (Klebsiella spp., E. coli, Pseudomonas and Acinetobacter, respectively). Also in the same study, when the agents were evaluated according to the foci of infection, the most frequently isolated microorganisms were Klebsiella spp. and Acinetobacter spp. in respiratory tract, Klebsiella spp. in bloodstream infections, E. coli and Klebsiella spp. in urinary system infections (7). When the literature is evaluated, it is seen that Acinetobacter spp. and Klebsiella spp. are in the first place, as in our study. Candida species are another cause of blood infections that are accepted as hospital infections in intensive care units. In studies performed, Candida species have been reported as an infectious agent in $8-15 \%$ of ICUs (3). We found the rate of Candida spp as $9.1 \%$ in our study.

The main limitation of our study is the fact that it is a retrospective study with a relatively small sample size, single center, and single ICU. Studies with more comprehensive analyses and a larger number of patients can provide further data on these variables.

\section{CONCLUSION}

The risk of HAI is high in patients hospitalized in ICUs. Mortality and morbidity increase in patients who develop nosocomial infections, and the length of hospital stay is prolonged. In order to control nosocomial infections, HAI incidences and rates should be evaluated, infectious agents and resistance profiles should be regularly monitored, and prospective effective infection control strategies should be developed by taking necessary precautions according to surveillance results. Taking these precautions plays an important role in reducing mortality and morbidity.

\section{ETHICAL DECLARATIONS}

Ethics Committee Approval: Approval for the study was granted by the Ethics Committee of Dicle University Faculty of Medicine (Date: 06.02.2020, Decision No: 144).

Informed Consent: Because the study was designed retrospectively, no written informed consent form was obtained from patients

Referee Evaluation Process: Externally peer-reviewed.

Conflict of Interest Statement: The authors have no conflicts of interest to declare.

Financial Disclosure: The authors declared that this study has received no financial support. 
Author Contributions: All of the authors declare that they have all participated in the design, execution, and analysis of the paper and that they have approved the final version.

\section{REFERENCES}

1. Bilge N, Yevgi R, Ceylan M, Parlak E, Şimşek F. Nosocomial infection rates of three-years in neurological intensive care unit and relationship to mortality. Anatolian Curr Med J 2021; 3; 158 64.

2. Mitharwal SM, Yaddanapudi S, Bhardwaj N, Gautam V, Biswal $\mathrm{M}$, Yaddanapudi L. Intensive care unit-acquired infections in a tertiary care hospital: An epidemiologic survey and influence on patient outcomes. Am J Infect Control 2016; 44: e113-7.

3. Samloglu P and. Atalay S. Five-year analysis of central nervous system, blood and nosocomial infection factors associated with central venous catheter in adult and pediatric intensive care units. J Biotechnol and Strategic Health Res 2021; 5: 119-124.

4. D'Agata EM, Mount DB, Thayer V, Schaffner W. Hospitalacquired infections among chronic hemodialysis patients. Am I Kidney Dis 2000; 35: 1083-8.

5. Horan TC, Andrus M, Dudeck MA. CDC/NHSN surveillance definition of health care-associated infection and criteria for specific types of infections in the acute care setting. Am J Infect Control 2008; 36: 309-32.

6. Tüfek A, Tekin R, Dal T, et al. Evaluation of hospital infections developing in intensive care unit during a decade and review of literatüre. Dicle Med J 2012; 39: 492-8.

7. Vincent JL, Sakr Y, Singer M, et al. Prevalence and outcomes of infection among patients in intensive care units in 2017. JAMA. 2020; 323: 1478-87.

8. Taş SS and Kahveci K. Surveillance of hospital infections in long-term intensive care unit and palliative care centre: a 3-year analysis. J Contemp Med 2018; 8: 55-9.

9. Senol A, Balin SÖ. Common infections in intensive care units, gram-negative microorganisms, antibiotic resistance. KSU Med J 2021; 16: 35-9.

10.Sahin AR, Yıldız BT, Aktemur A, Topal B, Nazik S, Ateş S. Evaluation of nosocomial infections in a neurological intensive care unit of a university hospital. J Contemp Med 2019; 9: 43-7.

11. Cetin S, Celik I, Artan C. Evaluation of microorganisms and antibiotic resistance profile isolated in intensive care unit patients. Turk J Intensive Care 2021; 19: 9-17.

12.Ulusal Sağlık Hizmeti, İlişkili Enfeksiyonlar Sürveyans Ağ (USHIESA) Haziran 2021.https://hsgm.saglik.gov.tr.

13. Koçer I, Zer Y, Karaoglan I. The clinic significance of urinary culture results for catheter- related urinary tract infection. Bozok Med J 2020; 10: 62-6.

14. Abbasi SH, Aftab RA, Chua SS. Risk factors associated with nosocomial infections among end stage renal disease patients undergoing hemodialysis: a systematic review. PLoS ONE 2020; 15: e0234376.

15. Tang LY, Chen JG. Economic impact of nosocomial infection in hemodialysis patients. Acta Medica Mediterranea 2016, 32: 617.

16.Ulusal Sağlık Hizmeti, İlişkili Enfeksiyonlar Sürveyans Ağ̊ (USHIESA) Etken Dağılımı ve Antibiyotik Direnç Raporu 2020. Haziran 2021. https://hsgm.saglik.gov.tr. 